\title{
Superior Cervical Ganglion
}

National Cancer Institute

\section{Source}

National Cancer Institute. Superior Cervical Ganglion. NCI Thesaurus. Code C92213.

A sympathetic gang lion located approximately at the first and second cervical vertebral levels. 